made. Visits to the emergency department per patient in the 90 days after support from the home treatment team was initiated compared with the 90 days before decreased (number of visits after support initiated, median (range): $1(0-6)$ $v$. before, 1 (0-8)). Hospital admissions per patient after support was initiated also decreased (after, $0(0-7) v$. before, $1(0-11))$, as did mean hospital stay per patient after support was initiated (after, $0(0-57)$ days $v$. before, $9(0-52)$ days). All differences are significant $(\alpha<0.01$, two-sided test, Wilcoxon signed-rank test). In a survey, patient satisfaction was high.

According to a survey among staff the establishment of the home teams led to a higher focus on and understanding of the patient's needs and abilities, but also increased interaction with next of kin. Home visits had also contributed to the identification of problems in transitions between hospital and home. Recognising these deficiencies contributed to valuable learning for improvement, as it allowed problems to be addressed at an earlier stage.

\section{Reflections on improvement in mental health}

RJC's 'quality as strategy' management principle is used in all improvement work across mental healthcare: access to treatment, patient involvement, reducing the need for hospital admission, organisational development of teams, and many others areas. Using this approach to improvement has led RJC to be recognised for its ongoing work with quality in healthcare and for its successful long-term, system-wide improvement. In several national comparisons over the years, the RJC stands out as one of the top healthcare systems in Sweden for clinical results and quality, patient satisfaction, public trust in the healthcare system and financial results.

In our experience, the improvement methods used for physical healthcare can be successfully employed in mental healthcare. This has also contributed to an insight that measurements of effects of improvement efforts are possible and necessary also in mental healthcare. The challenges that remain include: strategies to identify the patient's needs more accurately, difficulties in understanding how different healthcare professionals can contribute, language barriers - to be able to care for non-Swedish-speaking patients. We aim to further develop our culture of co-producing care across the whole system, including patients and next of kin in the co-design of all our improvement activities.

\section{Author contributions}

All four authors contributed to the conception of the article. A.R. and A.Ö. drafted the first versions of the article, all four authors then revised it and gave input to its final version. A.R. finalised the article, which was the finally approved by the other authors. All four authors agree to be accountable for all aspects of the work.

\section{Declaration of interest}

None.

ICMJE forms are in the supplementary material, available online at https://doi.org/10.1192/bji.2020.37.

\section{References}

1 Andersson-Gäre B, Neuhauser D. The health care quality journey of Jönköping County Council, Sweden. Q Manage Health Care 2007; 16: 2-9.

2 Övretveit J, Staines A. Sustained improvement? Findings from an independent case study of the Jönköping Quality Program. $Q$ Manage Health Care 2007; 16: 68-83.

3 Berwick DM, Nolan TW, Whittington J. The triple aim: care, health, and cost. Health Aff 2008; 27: 759-69.

4 Batalden M, Batalden P, Margolis P, Seid M, Armstrong G, Opipari-Arrigan L, et al Coproduction of healthcare service. BMJ Qual Saf 2016; 25: 509-17.

5 Bodenheimer T, Bojestig M, Henriks G. Making systemwide improvements in health care: lessons from Jönköping County, Sweden. Q Manage Health Care 2007; 16: 10-5.

6 Nelson EC, Batalden PB, Godfrey M. Quality by Design: A Clinical Microsystems Approach. John Wiley \& Sons, 2011.

7 Johnson S. Crisis resolution and home treatment teams: an evolving model. Adv Psychiatr Treat 2013; 19: 115-23.

8 Institute for Healthcare Improvement. Driver Diagram. Institute for Healthcare Improvement, 2020 (http://www.ihi.org/resources/ Pages/Tools/Driver-Diagram.aspx)

\title{
THEMATIC PAPER \\ Reducing the risk of patient suicide in Tuscany
}

\author{
Giulia Dagliana, ${ }^{1}$ Sara Albolino, ${ }^{2}$ Laura Belloni ${ }^{3}$ and Tommaso Bellandi ${ }^{4}$
}

${ }^{1} \mathrm{MSc}$, Safety and Quality Manager, Coordinator WHO Collaborating Centre in Human Factor and Communication for the Delivery of Safe and Quality Care, Florence, Italy

${ }^{2}$ PhD-EurErg, Director, Centre for Clinical Risk Management and Patient Safety, Florence, Italy

${ }^{3} \mathrm{MD}$, Psychiatrist, Director of the
Patient suicide is one of the most frequent incidents in healthcare facilities to be reported to the National Observatory of Sentinel Events in Italy. Despite national initiatives, in Tuscany potentially preventable patient suicides still occur in both acute and community care settings. We describe here an aggregated qualitative analysis of 14 patient suicides that took place in public health services between 2017 and 2018. We outline the methodology and results of an improvement action we enacted in the healthcare system that involved reviewing and reinforcing relevant managerial strategies and clinical activities, with the aim of reducing potentially preventable patient suicides. 
Centre for Relational Issues of the Teaching Hospital Careggi, Florence, Italy

${ }^{4} \mathrm{PhD}$, PhD-EurErg, Director, Patient Safety Unit of the Tuscany Northwest Tuscany, Italy. Email: tommasobellandi@ gmail.com

Keywords. Patient suicide; prevention; patient safety; reporting and learning; thematic analysis.

First received 31 Dec 2019 Final revision 2 May 2020 Accepted 3 Aug 2020

\section{doi:10.1192/bji.2020.43}

(c) The Authors 2020. Published by Cambridge University Press on behalf of the Royal College of

Psychiatrists. This is an Open

Access article, distributed unde

the terms of the Creative

Commons Attribution-

NonCommercial-ShareAlike

licence (http://creativecommons.

org/licenses/by-nc-sa/4.0/), which

org/licenses/by-nc-sa/4.0/), which
permits non-commercial re-use,

permits non-commercial re-use,
distribution, and reproduction in

any medium, provided the same

Creative Commons licence is

included and the original work is

properly cited. The written per-

mission of Cambridge University

Press must be obtained for com-

Press must be
mercial re-use.

\section{The problem of patient suicide in Italy and in Tuscany}

The Ministry of Health in Italy considers risk management to be a specific objective to be developed by national policies. The National Health Plan states that there is a need to guarantee and monitor the safety of healthcare and biomedical technology. To facilitate delivery of this objective a standardised computerised method has been introduced, which collects and analyses data on adverse events. Specific actions have been instituted to provide education and training for all stakeholders. This is conducted throughout the healthcare system, including governance, at national, regional and local levels. According to data from the National Observatory on Sentinel Events, patient suicide is the second most frequent type of event to be reported under the terms of the Plan, accounting for $15.4 \%$ of incidents over the period 2005 to 2012. ${ }^{1}$ Incidents reported include suicides and suicide attempts that occurred during the delivery of healthcare services in hospitals or out-patient facilities. In 2008 the Ministry of Health published a recommendation for the prevention of in-patient suicide, asking healthcare organisations and professionals to improve preventive actions. The recommendation provided for the training of healthcare workers, the organisation of services and improvements to the work environment. Also in the audits conducted by The Joint Commission, the physical environment of the inpatient unit in which the suicide occurred was the most important contributory factor in $84 \%$ of reported incidents. ${ }^{2}$ Despite the complexity of factors leading to patient suicide, the Ministry recognised that, when the event occurs during delivery of healthcare services, there would have been opportunities for prevention. Accordingly, recommendations were made for preventive measures to be taken, on the basis of evidence from research and professional consensus. $^{3}$
Within this framework sits the Centre for Clinical Risk Management and Patient Safety of the Tuscany Region (Centre GRC), which is tasked to implement safety solutions and prevent suicides within healthcare facilities. The Centre GRC is a World Health Organization Collaborating Centre with a remit to improve the safety and quality of care. It provides a regional clinical governance structure that promotes safety culture through a 'human factors and ergonomics' approach to managing complex systems. The Centre GRC operates on a shared vision of safety. The emphasis is on sharing experiences and includes field practices for safety that accord with operational reality; it aims to highlight effective and critical interventions.

The systemic approach of the Centre to improving patient safety is strengthened by incorporating multidisciplinary expertise that includes expertise in public health, safety and quality, industrial design, human factors, sociology, communication science and law. The Centre acts as regional hub for the coordination of all the activities related to safety and quality of care within a broader network of structures and professional risk managers at the level of individual facilities. The Centre has, by means of facilitating collaboration, been able to build an implementation-research project with the cooperation of front-line staff.

The Centre GRC collects the data on patient safety incidents by using an advanced reporting and learning web-based platform. The system is called 'SiGRC' and has been developed with the intention of collecting reports about adverse events submitted on a voluntary basis, directly from front-line health workers. These reports are analysed on the basis of a narrative of the adverse events, to discover latent themes and thereby provide a learning opportunity. ${ }^{4}$

In the SiGRC, adverse events are categorised according to the International Classification for Patient Safety, ${ }^{5}$ and contributing factors are categorised according to an adapted classification of

Table 1

Structured data about the patients' suicides $(n=6)$ and attempted suicides $(n=8)$ reported to SiGRC in 2017-2018

\begin{tabular}{|l|l|}
\hline Units involved & $\begin{array}{l}\text { Internal medicine (3 suicides), general surgery (1 suicide attempt), psychiatry } \\
\text { neurology (1 } 1 \text { suicicide; } 1 \text { suicide attempt), geriatrics (1 suicide attempt), } \\
\text { suicide attempt), orthopaedics (1 suicide attempt) }\end{array}$ \\
\hline $\begin{array}{l}\text { Method for suicide or } \\
\text { attempt }\end{array}$ & Jumping from a window (8 cases), hanging (2 cases), suffocation (2 cases), drug overdose (2 cases) \\
\hline Location & 5 suicides took place in a bathroom, 4 in bedroom, 4 in a communal space, 1 unknown \\
\hline Contributory factors & Patient characteristics (8 cases) \\
\cline { 2 - 3 } & Lack of, or failure to apply, guidelines, recommendations, clinical protocols (5 cases) \\
\cline { 2 - 3 } & Environmental hazards (5 cases) \\
\cline { 2 - 3 } & Poor communication between staff and between staff and patients (3 cases) \\
\cline { 2 - 3 } & Human factors of the professionals involved (1 case) \\
\cline { 2 - 2 } & Characteristics of medications (1 case) \\
\hline
\end{tabular}

a Psychiatry refers to a hospital service where voluntary and compulsory psychiatric treatments are carried out during hospitalisation. It also carries out consultancy activities for other hospital services. It is located inside the hospital facilities.

b Mental health refers to the first reference center for citizens with mental illness. It coordinates all interventions for the prevention, treatment and rehabilitation of citizens with psychiatric pathologies within the territory. 
- The introduction of an objective checklist to identify, at the point of admission, patients at risk of suicide would be valuable, because it would help to avoid the underestimation of risk factors.

- There should be constant observation of patients who have been admitted after a suicide attempt, whether or not they have a history of mental health problems, until they have been seen by a psychiatrist.

- There should be specific training for clinical staff who are potentially going to be in charge of patients at risk of suicide.

- Sufficient time should be dedicated to the handover between staff who are responsible for patients at risk, both at the time of a shift change and at the time of transition between services.

- Early identification of patients at risk and a better monitoring of behavioural signs and symptoms should be instituted throughout the continuum of care.

- There should be improved continuity of communication of risks between professionals and services.

- The design of in-patient units should permit clinical staff to maintain visual supervision of at-risk patients at all times, and physical hazards that might facilitate suicide should be removed or modified.

the London Protocol: ${ }^{6}$ (a) patient factors; (b) task and technology factors; (c) individual (staff) factors; (d) team factors; (e) work environment factors; (f) organisational and management factors; (g) institutional context factors.

Between 2007 and 2018, hospitals reported 48 suicides and 32 attempted suicides to the SiGRC and these events were analysed according to the specified criteria. The greatest proportion of suicides occurred in internal medicine units, where there were 14 incidents; psychiatric services reported 7 incidents and surgical services reported 6. The highest number of suicide attempts was registered among patients in penitentiary institutions (10 incidents), including 7 in mental health units and 3 in neurological units.

The national recommendation for prevention of risk has been part of the regional accreditation requirements since 2011. Despite this theoretical implementation of government policy, the incidence of similar suicides remained unchanged during the period of reporting (2007-2018). We therefore decided to perform an aggregated analysis of the sentinel events. Applying the methodology of systemic analysis and a human factors approach, we conducted a thematic analysis on data from six cases of completed suicide and eight cases of attempted suicide that occurred among in-patients at eight hospitals belonging to five public trusts between 2017 and 2018 .

The analysis was conducted by a multidisciplinary team comprising experts in human factors, patient safety personnel from the regional centre, members of the clinical teams with responsibility for patient care, as well as risk managers from the participating trusts. We collected the available reports on SiGRC and prepared a summary of the aggregated analysis, with a short timeline for each case and descriptive statistics as shown in Table 1. We compared our data with those recently published by the USA's Joint Commission for the Accreditation of Health Organizations, which has a similar reporting structure for patient sui$\operatorname{cides}^{7}$ and with a narrative review from the Mayo Clinic. $^{8}$

Once the analysis that had been conducted at a local level had been confirmed, the team reviewed and integrated improvement actions that were not only specific for each event, but were scalable and potentially transferable to other settings. The main recommendations that emerged from the analysis of the narrative are summarised in Box 1 .

\section{Discussion}

Our narrative analysis revealed that most of the suicides and suicide attempts were unrelated to previously identified psychiatric disease. Several people who were admitted after suicide attempts described having suffered from social and economic marginalisation and deprivation. We recommend greater cooperation between social services and primary health services, including mental health services, to identify people who are experiencing adverse socioeconomic issues. They would be the first and more immediate point of contact for the population at large and are in a better position than hospital staff to detect those at socioeconomic risk.

To provide support for the professionals involved in the care of patients at risk of suicide, the regional Centre for Relational Issues is providing a long-term training programme on multiprofessional management of at-risk patients and prevention. This initiative had its origins in clinics serving penitentiaries and is due to be expanded to reach community mental health services. Individual health service trusts are expected regularly to update their policies and procedures for the prevention of patient suicide. Regional centres will monitor their implementation, and a 'patient safety walkaround' (PSW) agenda is to be enacted in 2020. The PSWs have proven to be an effective method of encouraging change. They reinforce the alliance between front-line staff and management and provide a safe and free-from-blame opportunity to discuss organisational successes and failures. So far, there have been two PSWs reviewing the penitentiary systems of the region. They assessed potential improvements to safety and the quality of care delivered to imprisoned people with mental disorders. These reviews have led to positive results in terms of improvement in the delivery of assistance to prisoners at risk of engaging in suicidal behaviours.

\section{Author contributions}

All the authors contributed to the design and execution of the reported aggregated analysis of sentinel events. G.D. and T.B. wrote the draft paper; S.A. and L.B. revised and integrated the preliminary document. 


\section{Declaration of interest}

None.

ICMJE forms are in the supplementary material, available online at https://doi.org/10.1192/bji.2020.43.

\section{References}

1 Italian Ministry of Health. 5th Report on Sentinel Events Monitoring. Italian Ministry of Health, 2012. (http://www.salute. gov.it/imgs/C_17_pubblicazioni_2353_allegato.pdf).

2 Sakinofsky I. Preventing suicide among inpatients. Can J Psychiatry 2014; 59: 131-40.

3 Ministero della Salute. Raccomandazione $n^{\circ} 4$ : Prevenzione del suicidio di paziente in ospedale [Recommendation 4: Prevention of In-Patients Suicide]. Ministero della Salute, 2008. (http://www. salute.gov.it/imgs/C_17_pubblicazioni_592_allegato.pdf).
4 Bellandi T, Albolino S, Tomassini CR. How to create a safety culture in the healthcare system: the experience of the Tuscany Region. Theor Issues Ergon Sci 2007; 8: 495-507.

5 World Health Organization. Conceptual Framework for the International Classification for Patient Safety Version 1.1: Final Technical Report (WHO/IER/PSP/2010.2). World Health Organization, 2010.

6 Taylor-Adams S, Vincent C. Systems analysis of clinical incidents: the London Protocol. Clin Risk 2007; 10: 211-20.

7 Williams SC, Schmaltz SP, Castro GM, Baker DW. Incidence and method of suicide in hospitals in the United States. Jt Comm J Qual Patient Saf 2018; 44: 643-50.

8 Bostwick JM, Rackley SJ. Completed suicide in medical/surgical patients: who is at risk? Curr Psychiatry Rep 2007: 9: 242-6.

\section{THEMATIC PAPER}

\title{
Perinatal mental health around the world: priorities for research and service development in South America*
}

\author{
Marta B. Rondon ()
}

Consultant Psychiatrist, National Institute for Maternal and Perinatal Health (INMP), Lima Peru.Email: mbrondon@gmail. com

Conflicts of interest: None.

${ }^{*} \mathrm{~A}$ video abstract for this article is available at https://vimeo.com/ bjpsych/bji-2020-9.

\section{Keywords. South America; peri-} natal psychiatry; research priority; services.

First received 24 May 2019 Final revision 9 Jan 2020 Accepted 30 Jan 2020

\section{doi:10.1192/bji.2020.9}

(c) The Author 2020. This is an Open Access article, distributed under the terms of the Creative Commons Attribution licence (http://creativecommons.org/ licenses/by/4.0/), which permits unrestricted re-use, distribution, and reproduction in any medium provided the original work is properly cited.
Research on the topic of poor perinatal mental health in South America is scarce. Nevertheless, studies have shown that it is not uncommon, and that it is linked to women's experience of sexual and intimate partner violence and to inequality, poverty and low educational attainment. High-quality research in large samples with rigorous methodology is a priority, so that data from this region may be compared and analysed in systematic reviews. The links with intimate partner violence need to be explored. Risk and protective factors must be investigated with a strong intercultural perspective. Service integration needs to be implemented. This will require improvements in the availability, accessibility and quality of obstetric and mental health services. There is a need for targeted evidence-based interventions for women and children at risk that incorporate a strong gender and rights perspective.

Perinatal mental health is a modern concept that brings together newly acquired awareness of the relationship between the quality of care and nurturing in the early years of life and health in adulthood. Customarily based on developmental, attachment and systems theory frameworks, this concept encompasses the well-being of parents and infants from conception to 1 or 2 years of age. ${ }^{1}$ It is unfortunate that maternal mortality and extreme morbidity are still serious concerns in South America, despite promising rates of economic growth. In this context, the lack of prioritisation of the psychosocial and mental aspects of perinatal care is obvious, in spite of epidemiological evidence of the high prevalence of maternal perinatal affective disorders. ${ }^{2}$

\section{Regional prevalence of perinatal mental illness and associated risk factors}

As in other low- and middle-income countries (LMICs), perinatal affective disorders are more common in South America than in high-income countries. In 2012, Fisher and her group reviewed the literature on maternal pre- and postnatal mental health from 17 LMICs. ${ }^{3}$ They report a pooled prevalence of $15.9 \%$ (95\% CI 15.0 $16.8 \%$ ) for antenatal depression and $19.8 \%(95 \%$ CI 19.2-20.6) for postnatal depression. More recently, colleagues and I reviewed the literature and found a pooled prevalence for antenatal depression of $25.3 \%$ (95\% CI 21.4-29.6) from 51 papers (17 of which came from Latin America). ${ }^{4}$ The pooled prevalence rate for postpartum depression was $19.0 \%$ (95\% CI $15.5-23.0 \%)$ from 53 papers (11 from Latin America). Both reviews describe classic risk factors, such as previous depression history, obstetric complications and other illnesses concomitant with the pregnancy, poverty and low socioeconomic standing at the time of pregnancy, as would be expected. Furthermore, childhood maltreatment, exposure to intimate partner violence and pregnancy intendedness are identified as risk factors for perinatal mental illness.

Published rates of postpartum depression have been correlated with indices of national health 